\title{
Serviços de urgência e emergência: quais os motivos que levam o usuário aos pronto-atendimentos?
}

\author{
Ariane Bôlla Freire ${ }^{1}$, Débora da Luz Fernandes ${ }^{1}$, Juliana da Silva Moro², \\ Marindia Marques Kneipp ${ }^{3}$, Cleci Maria Cardoso ${ }^{4}$, Suzinara Beatriz Soares De Lima ${ }^{5}$
}

\begin{abstract}
RESUMO
Introdução: A superlotação dos Pronto-Atendimentos (PA) de Santa Maria - RS é um dos principais problemas do sistema de saúde local. Objetivo: Analisar os motivos da procura dos usuários pelos serviços de um Pronto-Atendimento no município de Santa Maria, RS. Metodologia: Levantamento de dados, método descritivo e analítico do tipo transversal. 0 estudo foi realizado no PA Flávio Schineider de maio a junho de 2013. Foi utilizado um questionário aplicado aos usuários do serviço que aborda dados pessoais e sobre a consulta. Resultados: A amostra constituiu-se de 180 pacientes (58 homens e 122 mulheres) na faixa etária de 17 a 60 anos. Sobre o motivo da consulta verificou-se a maior prevalência de febre. Referente à escolha do PA relataram-se situações de urgência e emergência e localização mais próxima da residência. Conclusão: Os entrevistados consideraram seus quadros clínicos uma situação de urgência e emergência, apesar da realidade do caso não ser avaliado uma situação de agravo à saúde.
\end{abstract}

Descritores: Saúde; SUS; Urgência; Emergência.

\section{Urgency and emergency services: what are the reasons that lead user to emergency care?}

\begin{abstract}
Introduction: The overcrowding of emergency unit of Santa Maria - RS is one of the main problems of the local health system. Objective: To analyze the reasons for the demand of users for the services of an emergency care in the city of Santa Maria, RS. Methodology: Survey data, descriptive and analytic of transverse type method. The study was conducted in emergency unit Flavio Schneider from may to june 2013. Was applied a questionnaire to the service users that addresses personal data and about of the consultation. Results: The sample consisted of 180 patients (58 men and 122 women) aged 17-60 years old. About the reason for the consultation there was a higher prevalence of fever. Concerning the choice of this emergency unit was reported urgency and emergency situations and the nearest house location. Conclusion: The interviewees considered their clinical condition an urgency and emergency situation, although the reality of the case not be assessed a situation of serious health hazard.
\end{abstract}

Descriptors: Health; UHS; Urgency; Emergency.

${ }^{1}$ Acadêmica de Fisioterapia na Universidade Federal de Santa Maria (UFSM), Santa Maria, RS, Brasil.

${ }^{2}$ Acadêmica de Odontologia na Universidade Federal de Santa Maria (UFSM), Santa Maria, RS, Brasil.

${ }^{3}$ Acadêmica de Farmácia na Universidade Federal de Santa Maria (UFSM), Santa Maria, RS, Brasil.

${ }^{4}$ Especialista em Saúde Pública pelo Centro Universitário Internacional (UNINTER), Santa Maria, RS, Brasil.

${ }^{5}$ Pós- doutora em Enfermagem pela Universidade Federal de Santa Catarina (UFSC), Florianopólis, SC, Brasil. 


\section{Introdução}

Em 1988, como marco da redemocratização do País, foi aprovada a nova Constituição Federal. ${ }^{1}$ Entre suas determinações, estabeleceram-se as bases para a edição da Lei 8080/90, que instituiu o Sistema Único de Saúde (SUS) ${ }^{2}$. A implantação do SUS é ainda um desafio que exige esforço intenso e solidário entre as esferas de governo, que envolve a Gestão do Sistema, os Conselhos de Saúde e os Recursos Humanos do setor. O empenho desses segmentos constitui a base para a viabilização e a implementação de ações e de serviços acolhedores, qualificados e resolutivos, e para permitir avanço no acesso e na inclusão de todos os cidadãos a esse sistema ${ }^{3}$.

A Legislação do SUS, embora não tenha tratado especificamente da atenção às urgências, definiu as competências de cada esfera de governo, delegando aos gestores, federal, estadual, municipal e aos respectivos conselhos de saúde a autonomia para a edição e aplicação de medidas, normas técnicas e estratégias visando a sua implantação e implementação, garantidas as diretrizes de universalidade e de igualdade de acesso e da integralidade de assistência ${ }^{4}$.

O cuidado em saúde no SUS foi ordenado em níveis de atenção, como a assistência básica, média e de alta complexidade, visando melhor programação e planejamento das ações e serviços do sistema de saúde ${ }^{5}$. Nesse sentido, as Unidades Básicas de Saúde (UBS) compõem a estrutura física básica de atendimento aos usuários do SUS e têm como principal fundamento ser a porta de entrada preferencial ao sistema de saúde. Quando funcionam adequadamente, a população consegue resolver a maioria dos seus problemas de saúde e, por conseguinte, podem reduzir as filas nos níveis de média e alta complexidade que correspondem às Unidades Básicas Distritais de Saúde (UBDS) e à assistência hospitalar de especialidades respectivamente ${ }^{6}$.

As UBDS têm-se interligadas as unidades de pronto-atendimento (PA), que são estruturas de complexidade intermediária entre as UBS e as portas de urgência hospitalares que compõem uma rede organizada de atenção às urgências. O PA constitui-se em um serviço organizado para funcionar 24 horas/dia ininterruptamente, atendendo qualquer pessoa que procure, sem agendamento prévio ou limite de consultas, com caráter de urgência ou emergência?.

Consideraram-se os conceitos de urgência e emergência sendo a ocorrência imprevista de agravo à saúde com ou sem risco potencial de vida, além da constatação médica de condições de agravo à saúde que impliquem em risco iminente de vida ${ }^{8}$. Em razão do crescente número de atendimentos, os pronto-atendimentos, embora tendo como alvo os serviços de urgência, acabam por atender usuários considerados não urgentes, resultando no aumento da demanda para casos que não seguem os critérios de urgência e emergência 5 .

É amplamente reconhecido que a procura por serviços de urgência e emergência para casos que poderiam ser resolvidos em serviços de menor densidade tecnológica, ocorre independente do nível de desenvolvimento econômico e social dos países. Observa-se que, apesar da progressiva expansão da rede básica e da implantação do Programa Saúde da Família, a demanda pelos serviços de urgência e emergência não vem se modificando. Constata-se divergência no modo de pensar e tratar a urgência pelos gestores, profissionais de saúde e usuários desses serviços. Apesar da disponibilidade de portas de entrada no sistema de saúde hierarquicamente estabelecidas pelos gestores e técnicos, verifica-se a busca da população pelos serviços de urgência, sem que fique clara a compreensão sobre os motivos dessa procura ${ }^{9}$.

O presente estudo resulta do projeto "Reestruturação da Rede de Atenção Às Urgências E Emergências" referente ao Programa de Educação Tutorial em Saúde - PET-SAÚDE. A preocupação em prestar serviços que estejam consoantes com as necessidades da população torna o Programa de Educação pelo Trabalho para a Saúde, PET-SAÚDE, uma estratégia para a qualificação via processo de integração, tanto dos serviços quanto dos futuros profissionais. Sendo assim, busca contribuir na formação de uma rede de suporte em urgência e emergência no município de Santa Maria e região de abrangência.

Assim como a maior parte dos serviços de emergência do país, a superlotação dos pronto-atendimentos de Santa Maria - RS é um dos principais problemas do sistema de saúde local, pois o deslocamento da população em direção a esse serviço promove o pronto-socorro a um prestador de atenção primária à saúde, e não, de fato, destinado ao atendimento de urgências e emergências.

Diante do exposto, o presente estudo justifica-se pela evidente importância de pesquisar quais os motivos da população procurar o PA, para a elaboração de políticas públicas de saúde que proporcionem qualificação do atendimento de urgência e emergência e um aumento do poder de resolução da atenção básica. Dessa forma, o objetivo deste estudo é analisar os motivos da procura dos usuários pelos serviços de um Pronto-Atendimento no município de Santa Maria. 


\section{Metodologia}

Esta pesquisa é do tipo levantamento de dados utilizando um método descritivo e analítico do tipo transversal e aborda dados do projeto "Reestruturação da Rede de Atenção Às Urgências E Emergências".

A pesquisa foi conduzida de acordo com os padrões éticos exigidos. Após a aprovação do projeto no Comitê de Ética e Pesquisa (CEP) da UFSM, sob número do parecer 250.561, os Termos de Consentimento Livre e Esclarecido (TCLE) foram levados ao Pronto-Atendimento e, posteriormente foram entregues aos sujeitos e explicados sobre os riscos e os benefícios da pesquisa e sobre o direito de autonomia dos usuários submetidos ao estudo no local. Quanto aos riscos a pesquisa não produz nenhum tipo, porém, caso ao responder as questões o indivíduo sentir algum tipo de desconforto emocional, será encaminhado para o serviço de psicologia da UFSM. Referente aos benefícios, o projeto não trará diretamente nenhum beneficio ao respondente e sim para o sistema de saúde do município e para os serviços de urgência e emergência.

O estudo foi realizado no período de maio a junho de 2013, no Pronto-Atendimento Flávio Schineider, o qual atende pelo SUS. A amostra constituiu-se de 180 pacientes (58 homens e 122 mulheres) na faixa etária de 17 a 60 anos, que foram atendidos no serviço. $O$ instrumento utilizado neste estudo para a coleta de informações foi um questionário aplicado aos usuários do SUS contendo 30 questões que abordam dados pessoais e dados sobre a consulta.

Os dados coletados do questionário pelos pesquisadores foram armazenados e tabulados em ficha própria. No presente estudo serão apresentados dados que caracterizam o motivo da consulta e da escolha do respectivo Pronto-Atendimento Flávio Schineider.

\section{Resultados e discussão}

São apresentados inicialmente dados relativos ao motivo da consulta. Dessa forma, verificou-se a maior freqüência de atendimentos de pacientes que apresentavam febre (50) (27,77\%). Dos usuários entrevistados, $24,44 \%$ (44) relataram outro motivo agudo como causa da consulta; $13,33 \%$ (25) sintomas gastrointestinais; $12,22 \%$ (22) outro motivo não agudo, como mal-estar, tosse, febrícula, reações alérgicas controladas, entre outros; $7,22 \%$ (13) dor crônica agudizada; $6,66 \%$ (12) traumas; $5,59 \%$ (9) consulta de rotina e 2,77\% (5) relataram eventos associados à hipertensão arterial sistêmica (HAS), diabetes ou insuficiência cardíaca como motivo da consulta.

A figura 1 mostra a distribuição dos usuários que procuraram o PA conforme o motivo da consulta.

Figura 1 - Motivo da consulta no Pronto-Atendimento Flávio Schineider.

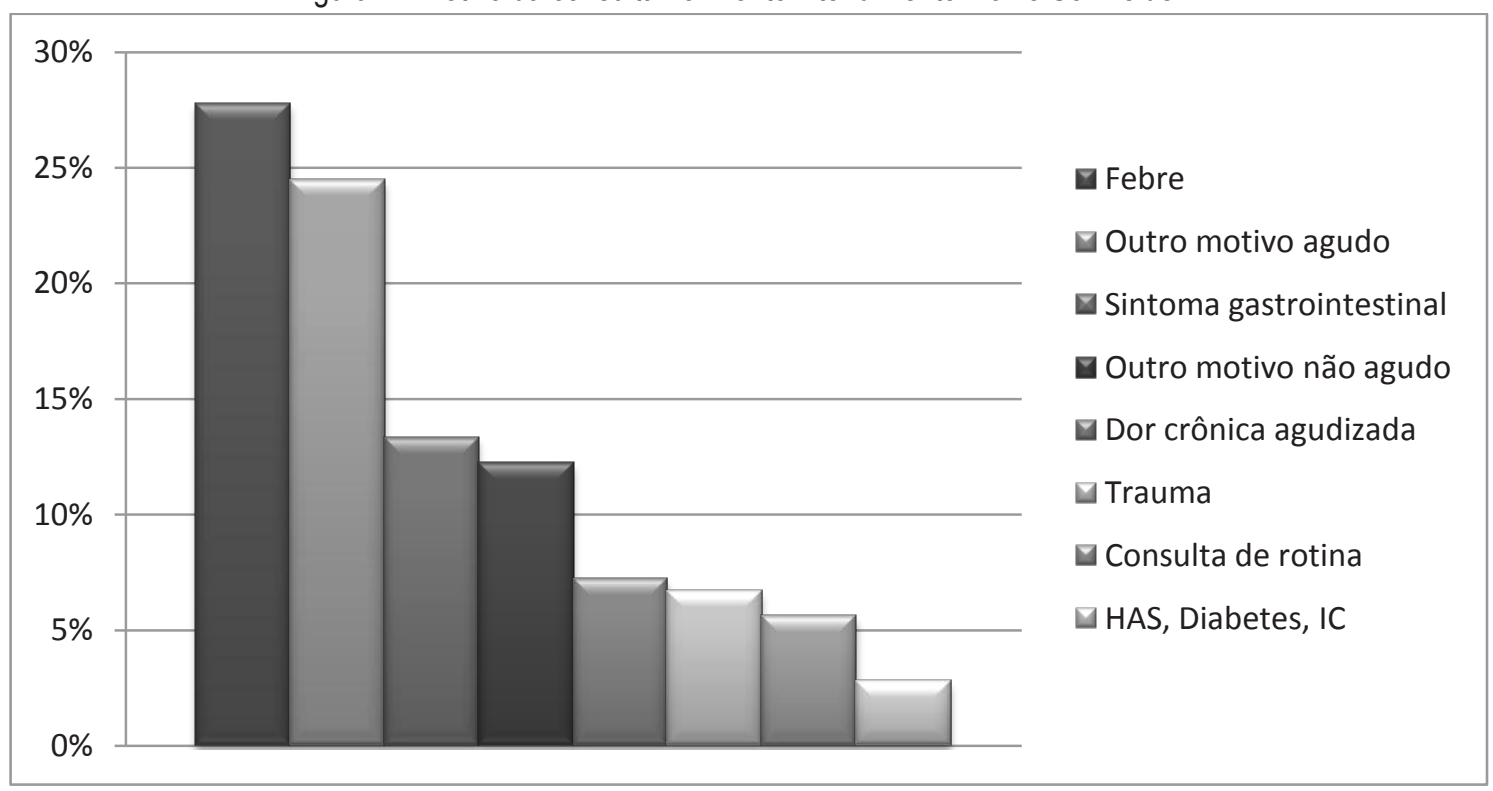

A partir dos resultados, percebe-se que o motivo mais prevalente de procura pelo PA foram indivíduos que apresentavam febre, seguidos de outros motivos agudos, como dor de garganta, dor no peito, coluna, entre outros. Diante disso, é possível constatar que a maior parte dos indivíduos considera seu quadro clínico como sendo situação de urgência e emergência, apesar do motivo predominante da consulta no Pronto-Atendimento não ser compatível com uma situação de agravo à saúde. 
Estes achados são semelhantes ao estudo de Souza et al. (2012), no qual os resultados demonstraram que o PA atende a prontidões e também atende aos usuários considerados não urgentes, resultando no aumento da demanda para casos que não seguem os critérios de urgência e emergência ${ }^{9}$. Há mais de uma década observam-se prontos-socorros lotados em razão de um deslocamento da população em direção a esses serviços, configurando-os como prestadores de atenção primária de assistência à saúde, não de fato destinados a atendimentos emergenciais ${ }^{10}$.

Olivat et al (2010) em seu estudo, verificou que as principais queixas relatadas pelos pacientes nos atendimentos dos Serviços do Pronto-Socorro Municipal de Capão Bonito (SP) foram agudas e desconforto físicos, estas são consideradas não urgentes e seu processo terapêutico se enquadra nos serviços de atenção básica ${ }^{11}$. Pires et al (2013) observou 0 mesmo motivo da procura pelo serviços de urgência e emergência dos usuários do PA de Belo Horizonte, das quais são motivados por enfermidades leves que podem ser resolvidas nas Unidades Básicas de Saúde ${ }^{12}$.

Referente ao motivo da escolha do PA, considerando que cada participante poderia considerar mais de uma alternativa, os motivos da procura ao PA Flávio Schineider foram: situação de urgência e emergência (48 votos); localização mais próxima da residência ou local de trabalho (48 votos); horário de atendimento mais acessível (47 votos); atendimento efetivo e resolutivo (23 votos); na UBS não havia médico para atendimento (22 votos); exames realizados com agilidade (19 votos); na UBS não havia ficha para atendimento (17 votos); atendimento rápido (14 votos); outro motivo (12 votos); instalação mais agradável e equipada (7 votos).

A tabela 1 demonstra os dados relativos ao motivo da escolha do PA.

Tabela 1 - Motivo da escolha do Pronto-Atendimento Flávio Schineider.

\begin{tabular}{c|c}
\hline Motivo da escolha do Pronto Atendimento & N \\
\hline Situação de urgência e emergência & 48 \\
\hline Localização próxima da residência & 48 \\
\hline Horário de atendimento acessível & 47 \\
\hline Atendimento efetivo e resolutivo & 23 \\
\hline Falta de médico na UBS & 22 \\
\hline Exame realizado com agilidade & 19 \\
\hline Falta de ficha na UBS & 17 \\
\hline Atendimento rápido & 14 \\
\hline Outro motivo & 12 \\
\hline Instalação rápida e agradável & 7 \\
\hline
\end{tabular}

A procura por serviços de saúde envolve fatores que, dependendo do modo como são ordenados, definirão a escolha pelo usuário. Assim como foi visto em relatos de pacientes através deste estudo, são influentes a gravidade ou a urgência do problema/necessidade, tecnologia disponível, resolutividade da atenção, acolhida, condições de acesso, agilidade no atendimento, experiências vividas pelo paciente ou sua família, destreza na marcação de exames ou encaminhamentos para outros serviços, localização, bem como o vínculo estabelecido pelo usuário com profissionais, serviços e o sistema de saúde ${ }^{13}$.

Diversas foram as justificativas para a procura pelo PA, no entanto, dentre elas, as potencialidades do PA na forma de alta densidade tecnológica, uma vez que na maioria das condutas está a solicitação de exames e prescrição de medicamentos que são ali mesmo administrados, a fim de atender às necessidades dos usuários que o procuram 5 .

Em razão da oferta restrita de serviços, o público excedente procura atendimento em locais que concentrem maior possibilidade de portas de entrada, e os PA's e emergências hospitalares correspondem ao perfil de atender às demandas de forma mais ágil e concentrada. Apesar de superlotados, impessoais e atuando sobre a queixa principal, esses locais reúnem um somatório de recursos, como consultas, remédios, procedimentos, exames laboratoriais e internações, enquanto as unidades de saúde da atenção básica oferecem apenas a consulta médica ${ }^{14}$, fato que também foi observado nesta pesquisa.

A prescrição de medicações, sob a forma de conduta, reforça o modelo de atenção baseado na queixa-conduta dos casos agudos, buscando resolver as necessidades de saúde trazidas pelos usuários. Portanto, são condutas de resolução em curto prazo, fato que explica os retornos subsequentes dos mesmos usuários ao PA, ou seja, quando retornam os sintomas, novamente, os usuários procuram atendimento ${ }^{5}$.

Dessa maneira, como objetivo de organizar a demanda e estabelecer prioridades para o atendimento nesses serviços, uma estratégia proposta é o acolhimento com classificação de risco, que permite a priorização do atendimento aos 
pacientes com doenças graves ou com instabilidade de órgãos/sistemas, otimizando recursos, melhorando a qualidade da assistência, orientando o usuário para a resposta mais adequada à sua demanda naquele momento ${ }^{15}$.

Ademais, deve ser observado e analisado a real necessidade de cada indivíduo, identificando as principais razões de saúde que geram a busca do atendimento. Ainda, é de grande importância que haja a possibilidade de um indivíduo que acredita ter um problema de saúde consultar um profissional que possa ajudá-lo a entender se o problema gera risco iminente a sua saúde o suficiente para necessitar de atenção adicional, ou se é um problema auto-limitado que não necessita de cuidado emergencial ${ }^{16}$.

Segundo estudo de Tomasi et al. (2011), necessita-se o desenvolvimento de alternativas criativas para que sejam supridas as limitações e aproximar-se de um modelo mais ideal e efetivo, ajustado às necessidades da população de cada região e a capacidade de oferta do sistema ${ }^{17}$.

\section{Considerações Finais}

Com base nos resultados encontrados no presente estudo, na maioria dos casos, os sujeitos da pesquisa declararam a escolha do PA por considerarem seus quadros clínicos como situação de urgência e emergência, apesar da realidade do caso clínico não ser considerado uma situação de agravo à saúde com risco iminente de vida. Ainda, os usuários descreveram como principais motivos da escolha do PAa localização mais próxima da residência ou local de trabalho, horário de atendimento mais acessivel e de forma efetiva e resolutiva, além de relatarem falta de médico na UBS para atendimento.

De acordo com o pressuposto de que a demanda aumentada no PA de casos não urgentes pode trazer dificuldades para a equipe, a fim de proporcionar um atendimento mais acolhedor às necessidades da população entende-se a importância dos gestores e gerentes de saúde em ampliar as ofertas de serviços da atenção básica que atendam os usuários em suas inúmeras queixas.

\section{Referências Bibliográficas}

1. Brasil. Constituição da República Federativa do Brasil. Seção II da Saúde. Senado, Brasília, DF,1988.

2. Santos JS, Scarpelini S, Brasileiro SLL, Ferraz CA, Dallora AELV, Sá MFS. Avaliação do modelo de organização da Unidade de Emergência do HCFMRP-USP, adotando, como referência, as políticas nacionais de atenção às urgências e de humanização. Medicina (Ribeirão Preto). 2003; 36: 498-515.

3. Santa Maria. Secretaria Municipal de Saúde. Plano Municipal de Saúde 2009-2012. Santa Maria, 2008.

4. Brasil. Lei no 8080/90, de 19 de setembro de 1990. Dispõe sobre as condições para a promoção, proteção e recuperação da saúde, a organização e o funcionamento dos serviços correspondentes e dá outras providências. Brasília, DF, 1990.

5. Gomide MF, Pinto IC, Gomide DMP, Zacharias FCM. Perfil de usuários em um serviço de pronto atendimento. Medicina (Ribeirão Preto). 2012; 45(1): 31-8.

6. Brasil. Ministério da Saúde. O SUS de A à Z. Garantindo saúde nos municípios. $3^{0}$.ed. Brasília, MS; 2009.

7. Pinto IC, Rodolpho F, Oliveira MM. INTO, I.C. Pronto-atendimento: a percepção da equipe de Enfermagem quanto ao seu trabalho no setor de recepção. Rev Gaúch Enferm (Porto Alegre). 2004; 25: 81-8.

8. Conselho Regional de Medicina. 1988. Disponível em: <http://www.cremesp. org.br/clippings/legislacao_anteriores_ integra.php>. Acesso em: 20 mai 2014.

9. Rati RMS, Goulart LMHF, Alvim CG. Criança não pode esperar: a busca de serviço de urgência e emergência por mães e suas crianças em condições não urgentes, Ciênc Saúde Coletiva (Rio de Janeiro). 2013; 18 (12): 3663-72.

10. Barakat SFC. Caracterização da demanda do Serviço de Emergências Clínicas de um hospital terciário do município de São Paulo. [Tese de Doutorado]. São Paulo. Faculdade de Medicina, Universidade de São Paulo. 2009.

11. Olivat FN, Brandão GAM, Vazquez FL, Paranhos LR, Pereira AC. Perfil da demanda de um pronto-socorro em um município do interior do estado de São Paulo. RFO (Passo Fundo). 2010; 15(3): 247-52.

12. Pires MRGM, Göttems LBD, Cupertino TV, Leite LS, Vale LRD, Castro MAD, et al. A utilização dos serviços de atenção básica e de urgência no sus de belo horizonte: problema de saúde, procedimentos e escolha dos serviços. Saúde Soc (São Paulo). 2013; 22(1): 211- 22. 
13. Traverso-Yépes M, Morais NA. Reivindicando a subjetividade dos usuários da rede básica de saúde: para uma humanização do atendimento. Cad Saúde Pública (Rio de Janeiro). 2004; 20(1): 80-8.

14. Marques CQ, Lima MADS. Demandas de usuários a um serviço de pronto atendimento e seu acolhimento ao sistema de saúde. Rev Latino-Am Enferm. (Ribeirão Preto). 2007; 15(1).

15. Brasil, Ministério da Saúde. Secretaria de assistência à saúde. Núcleo técnico da política nacional de humanização. Acolhimento com classificação de risco. Brasília: Ministério da Saúde, 2004.

16. Brasil. Ministério da Saúde. Secretaria de Atenção à Saúde. Departamento de Atenção Básica. Atenção primária: equilíbrio entre necessidades de saúde, serviços e tecnologia. Brasília: Unesco, 2002.

17. Tomasi E, Facchini LA, Thumé E, Piccini RX, Osorio A, Silveira DSD, et al. Características da utilização de serviços de atenção básica à saúde nas regiões Sul e Nordeste do Brasil: diferenças por modelo de atenção. Ciênc Saúde Coletiva (Rio de Janeiro). 2011; 16(11): 4395-4404.

\section{Ariane Bôlla Freire}

Endereço para correspondência - Rua: João Batista da Cruz Jobim, n² 235, Bairro: Medianeira, CEP: 97060-330, Cidade: Santa Maria, RS, Brasil.

E-mail: aribfreire@hotmail.com

Lattes: http://lattes.cnpq.br/5200221092017780

Débora da Luz Fernandes - deboradaluzfernandes@yahoo.com.br

Juliana da Silva Moro - juliana.moroo@hotmail.com

Marindia Marques Kneipp - marindia_mk@hotmail.com

Cleci Maria Cardoso - cleci.m@hotmail.com

Suzinara Beatriz Soares De Lima - suzibslima@yahoo.com.br

\section{Enviado em 04 de agosto de 2014.} Aceito em 19 de março de 2015. 